\title{
Die Zukunft ist digital
}

\section{B. Eibinger-Miedl}

Online publiziert am 27. Oktober 2017

(C) Springer-Verlag GmbH Austria 2017

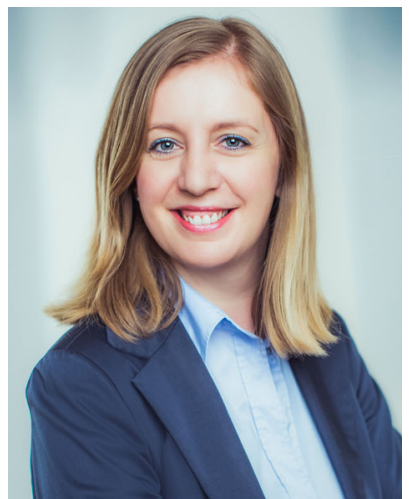

MMag. Barbara Eibinger-Miedl
Das Auto, das selbst fährt. Der Kühlschrank, der die Milch selbst bestellt, bevor sie ausgeht. Die Fabrik, die computergesteuert nicht nur große Mengen, sondern auch kleinste Serien fertigen kann. Viele Dinge, die vor zehn Jahren noch utopisch klangen, sind heute bereits gelebte Realität. Denken wir nur an das Smartphone, das es vor elf Jahren noch nicht einmal gegeben hat und das heute unseren Alltag bestimmt: Wir steuern damit unsere Heizung, erledigen einen großen Teil unserer Büroarbeit oder unsere Bankangelegenheiten und haben unsere Lieblingsmusik, Fotos, Videos und Spiele immer bei uns.

Diese Beispiele zeigen eindrucksvoll: Wir sind mitten drin im digitalen Zeitalter. Und die Digitalisierung wird unsere Welt und unser Leben in den nächsten Jahren noch viel stärker verändern als wir es uns heute vorstellen können. Man kann dazu stehen, wie man will - eines steht jedenfalls fest: Der Prozess ist nicht aufzuhalten. Die Digitalisierung verändert auch alle Sektoren der Wirtschaft: Produktionsabläufe ändern sich, es entstehen neue Geschäftsmodelle und Berufe.

Für ein Innovations- und Forschungsland wie die Steiermark bietet die Digitalisierung große Chancen für wirtschaftliches Wachstum und damit für eine Steigerung der Lebensqualität in unserem Land. Im Wirtschaftsressort des Landes setzen wir daher auf die Digitalisierung und unterstützen unsere Unternehmen dabei, diese Chancen zu nutzen. Auch in der Wirtschaftsstrategie Steiermark 2025 ist die Digitalisierung ein wesentlicher Schwerpunkt.

Neben dem flächendeckenden Breitbandausbau im ganzen Land setzen wir vor allem auf drei Bereiche:

Weil Konjunktur immer im Kopf beginnt, schaffen wir Bewusstsein für die aktuellen Entwicklungen bei unseren Unternehmen und vermitteln Know-how für die Veränderungsprozesse und Geschäftsmodelle von morgen. Kürzlich ist die 50. Auflage des Digitaldialogs in Graz über die Bühne gegangen, bei dem wir Unternehmen und Forschungseinrichtungen auf die Entwicklungen im Bereich digitaler Technologien aufmerksam machen. Auch beim Zukunftstag der Steirischen Wirtschaft und dem „Fast Forward Award", dem Wirtschaftspreis des Landes Steiermark, wird den Bereichen "Smart Production" und "Smart Services" besonders Rechnung getragen.

Digitalisierung bedeutet auch Investitionen - in Menschen, Maschinen und Märkte. Mit der Digitalisierungsoffensive der Steirischen Wirtschaftsförderung SFG haben wir die Förderungsprogramme den künftigen Anforderungen angepasst. Der digitalisierte Arbeitsalltag erfordert zum Teil gänzlich neue Kompetenzen von Mitarbeiterinnen und Mitarbeitern. Dazu zählen Fertigkeiten im Umgang mit neuen Technologien ebenso wie persönliche Fähigkeiten - beispielsweise von Führungskräften, die mit virtuellen Teams in einer globalen Welt arbeiten. Deshalb unterstützen wir Qualifizierungsmaßnahmen für „Digital Skills" mit der Förderungsaktion „Erfolgs!Kurs”.

Mit der Innovationsförderung "Ideen!Reich" fördern wir Unternehmen, die neue Produkte und Dienstleistungen entwickeln und diese erfolgreich auf Märkten im In- und Ausland positionieren. In diesem Programm werden Projekte zum Thema Digitalisierung besonders hoch bewertet.

Die Nahversorgungsförderung „Lebens! Nah“ wurde ebenfalls um den digitalen Aspekt erweitert: Die Nahversorger in den steirischen Regionen werden bei der Erstellung innovativer Medien, der Nutzung neuer Plattformen und sozialer Netzwerke oder der Digitalisierung ganzer Geschäftsprozesse finanziell unterstützt.

Drittes Standbein der steirischen Digitalisierungsoffensive ist die Vernetzung von Wissenschaft und Wirtschaft: Bereits jetzt arbeiten Unternehmen und Forschungseinrichtungen in den steirischen Kompetenzzentren erfolgreich zusammen. Die Steiermark ist an $25 \mathrm{der}$ österreichweit 44 Kompetenzzentren beteiligt, 19 haben ihren Sitz in der Steiermark. Die Kompetenzzentren tragen wesentlich dazu bei, dass die Steiermark mit einer F\&E-Quote von 5,16 Prozent das Forschungsland Nummer eins in Österreich ist und auch in Europa an der Spitze steht.

Die Steiermark ist seit Jahrzehnten dafür bekannt, grenzüberschreitend zusammenzuarbeiten. Da auch die Digitalisierung keine Grenzen kennt, wurde mit "Silicon Alps" vor einem Jahr der erste länderübergreifende Cluster Österreichs ins Leben gerufen. Gemeinsam mit dem Land Kärnten, der Kärntner Wirtschaftsförderung und der Industrie haben wir dieses Netzwerk im Bereich Mikroelektronik - einem Stärkefeld der steirischen Wirtschaft - etabliert, dem nun bereits mehr als 70 Unternehmen und Forschungseinrichtungen angehören - darunter Global Player wie Infineon, AT\&S, ams, EPCOS oder NXP.

Kein Zufall also, dass die Steiermark auch den Zuschlag für das Headquarter des Forschungszentrums "Silicon Austria” bekommen hat: 200 Forscherinnen und Forscher werden künftig in Graz - gemeinsam mit Kolleginnen und Kollegen in Villach und Linz - wegweisende Ideen und Systeme in der Mikroelektronik entwickeln. Knapp 300 Millionen Euro investieren Bund, Länder und die Industrie in dieses Zukunftsfeld mit dem Ziel, Österreich zu einem weltweit führenden Land in der Mikroelektronik zu machen. Denn rund ein Zehntel der weltweiten Wirtschaftsleistung hängt direkt oder indirekt von Elektronikprodukten ab. In Österreichs Elektronikindustrie arbeiten mehr als 63.000 Menschen in über 180 Unternehmen und erwirtschaften einen Umsatz von rund 80 Milliarden Euro. Sie alle wollen und werden von Österreich aus die neue, digitale Welt mitgestalten!

Eibinger-Miedl, Barbara, Landesrätin für Wirtschaft, Tourismus, Europa, Wissenschaft und Forschung, Nikolaiplatz 3,8020 Graz, Österreich

(E-Mail: markus.poleschinski@stmk.gv.at) 